may be quite complicated, and that it is perhaps premature to assume that the new band of absorption appearing at $247 \mu \mu$ in irradiated ergosterol is a characteristic band of vitamin $D$.

The arrangement described here may prove to be useful in the study of other photochemical processes.

My best thanks are due to Prof. Farrington Daniels for the hospitality of his laboratory.

Stanislaw KaZIMIERz KoN.

Laboratory of Physical Chemistry, University of Wisconsin,

Madison, Wisconsin, July 13.

\section{The Stark Effect at Very High Field.}

For the spectroscopic study of atomic and molecular structure, the production of high magnetic and electric fields is of prime importance. I have lately been engaged in this problem, and although $I$ do not believe the limit has been reached, still, so far, I have obtained a field of $650 \mathrm{k} . \mathrm{v}$. per cm. (being roughly extrapolated from the previous date for $H_{a}$, neglecting the third and higher order Stark effect).

The tube used was a quartz one of the Lo Surdo
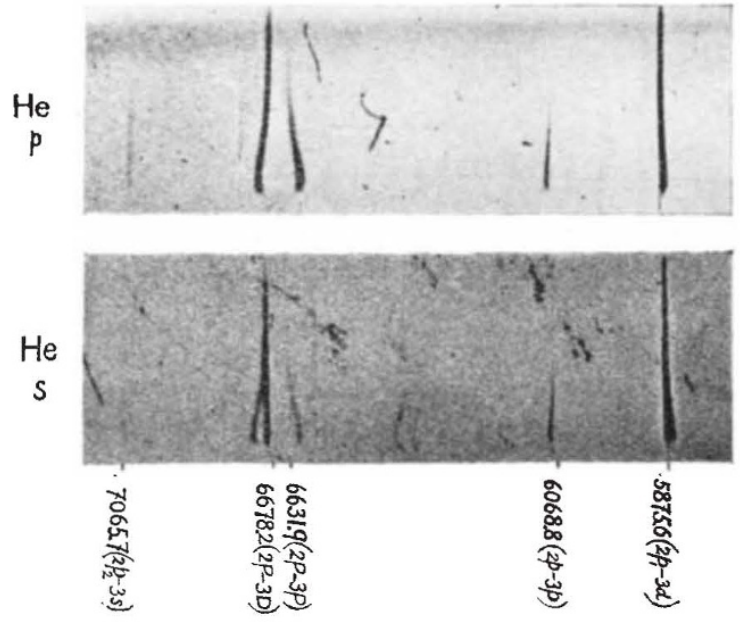

$$
\begin{aligned}
& \therefore \text { He }\left\{\begin{array}{l}
p: 580 \mathrm{KV} . / \mathrm{cm} . \text { extrapolated from } \mathrm{H}_{\beta} s \\
\text { S: } 625 \mathrm{KV} . / \mathrm{cm} . "
\end{array}\right. \\
& H \alpha \\
& \begin{array}{c}
H \alpha \\
p
\end{array} \\
& \bigwedge
\end{aligned}
$$

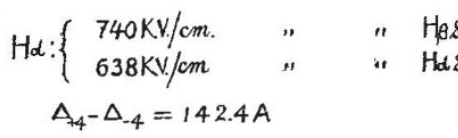

type, the inner diameter being 0.8 to $2 \mathrm{~mm}$. For the excitation, a powerful transformer with a synchronous motor rectifier was used. Particular care was taken to choose the most suitable phase setting of the vanes of the rectifier. In most cases the current remained below 0.5 milliamp. The exact value of the terminal voltage has so far not been measured; but, roughly speaking, the largest field would have been only of the order of $150 \mathrm{k} . \mathrm{v}$., if the ordinary positions of the vanes had been used.

With the help of this very strong field, I have observed various lines of the helium and lithium spectra which were hitherto not found to be affected by the electric field; for example, both the helium line $\lambda 7066 \mathrm{~A}$. and the lithium line $\lambda 6702 \mathrm{~A}$. were found to be shifted to the violet. In the case of the neon spectrum a large number of lines were found to show quite complicated patterns of the Stark effect, allowing a more extensive study than was carried out by Nyquist (Phys. Rev., 10, 226-243; 1917). With hydrogen an interesting feature observed was that the relative intensities of the $p$-component of $\mathrm{H}_{\alpha}$-line were found to be in the ratio $22: 65: 45$, instead of Stark's former data of $1: 1 \cdot 1: 1 \cdot 2$, and this new result seems to be in better agreement with Schrödinger's recent ealculations. The accompany. ing photograph (Fig. 1) shows a part of the $p$ - and $s$-components of the helium spectrum and the $p$ component of $\mathrm{H}_{\alpha}$. The discrepancy between the field values extrapolated from $\mathrm{H}_{\alpha}$ and $\mathrm{H}_{\beta}$ must be largely due to the third and higher order Stark effect of $\mathrm{H}_{\beta}$. The detailed report will be published in the scientific papers of the Institute.

In conclusion, I may state that it seems to be quite promising to apply this method for the Stark effect study of other elements.

The Institute of Physical and

Chemical Research, Tokyo, Japan.

\section{Gontractions for Titles of Periodicals.}

THE advantage of a standard list of contractions for periodical titles for general use in scientific journals would so outweigh initial inconveniences of changing present systems that it seems ungracious, with a comprehensive list of abbreviations now available in the "World List of Scientific Periodicals" (1901-1920), to question its suitability for general adoption. I am, however, doubtful whether the "World List". meets the particular requirements of journals circulating widely abroad. For these the title contractions must be free from ambiguities, indicative of the language of the originals, and full enough for a reader of any nationality to reconstruct the title sufficiently to enable him to find the titleentry easily in an alphabetically arranged catalogue of periodicals.

To secure freedom from ambiguity the use of the same contraction for words of different meaning should be avoided. The "World List" does not always do this. For example, Indian Engineering is contracted to Ind. Engng., and Journal of Industrial Hygiene to $J$. Ind. Hyg. A reader recognising the Ind. in J. Ind. Hyg. as standing for Industrial, might well assume that Ind. Engng. stood for Industrial Engineering, only to discover on obtaining the latter journal from a library or bookseller that it was not the one required.

As indicative of the language of the original the retention of an article, preposition, or conjunction in contractions of foreign titles is helpful. The "World List" normally omits these. The Annali di Clinica medica, Palermo, becomes simply Ann. Clin. med, being determined as Italian because of the higher order of familiarity of the Italian language over Portuguese, and because its form of contraction precludes English, French, or German. But the knowledge of languages implied here will not always save a reader from doubt. The French publication L'Argus : Journal International des Assurances, Paris, becomes simply Argus, which gives no clue to the language of the original, and a reader cannot be presumed to know that France alone has a scientific periodical of this name. The retention of the article, or of the imprint 'Paris' here seems necessary.

The third requirement, that the contraction be full enough to facilitate the reader's search for the journal-title in any library list, seems to me the most 